\title{
MODELLING THE BEHAVIOR OF SYSTEMS: \\ BASIC CONCEPTS AND ALGORITHMS
}

\author{
Tommaso Cotroneo \\ Mathematics Institute, \\ University of Groningen, \\ P.O. Box 800, 9700 AV Groningen, The Netherlands. \\ T.Cotroneo@math.rug.nl
}

Jan C. Willems

As above

J.C.Willems@math.rug.nl

\begin{abstract}
In this paper we introduce the behavioral approach as a mathematical language for describing dynamical systems, in particular systems modeled by high order constant coefficient linear differential equations. We investigate what data have to be added in order to express the influence of the environment and the initial conditions on the system. We give an algorithm to check whether these additional constraints are satisfied by a (unique) trajectory. We define the concepts of observability and controllability, and present algorithms which provide a constructive verification of such properties.
\end{abstract}

\section{INTRODUCTION}

The purpose of this paper is two-fold. Firstly, we introduce some of the main ideas of behavioral systems theory as an abstract framework for modeling and analysis of dynamical systems. Secondly, we show how algorithms can be developed which allow the analysis of system properties, even at the high level of generality at which we shall be working.

The starting point of our discussion is the definition of a system as the set of feasible trajectories of the variables whose dynamics we are modeling. Such a definition has two crucial aspects: on the one hand

The original version of this chapter was revised: The copyright line was incorrect. This has been corrected. The Erratum to this chapter is available at DOI: 10.1007/978-0-387-35514-6_15 
it abandons the idea of a system as an input/output map, i.e. as a signal processor, and simply makes it a relation between variables which evolve in time, but for which no hierarchical or cause/effect structure is given a priori. On the other hand, it distinguishes clearly between the system (i.e. the feasible trajectories) and its representations (e.g. equations, graphs, grammar rules, etc.).

Crucial definitions such as controllability and observability are also given in a representation-free fashion.

At the level of representations, we concentrate on linear differential systems, i.e. systems whose trajectories can be described as solutions to a set of linear differential equations. For such systems we describe algorithms which allow one to check controllability and observability, and which allow one to verify whether trajectories satisfying given constraints can be simulated.

One crucial issue we shall overlook for lack of space is that of describing systems as interconnections of smaller subsystems. Such a concept, very typical in engineering thinking, finds in the behavioral framework a nice formal description. It also motivates the introduction of the concept of latent variables, namely variables which we are not interested in modeling, but which we have to take into account, in order to describe the subsystems and the interconnections that provide the final model.

The main references to the ideas we will discuss are $[7,9]$; in [10], control issues are also addressed from this point of view; finally, $[8,6]$ use this same framework to study properties of systems described by partial difference and differential equations.

\section{MODELING A DYNAMICAL SYSTEM}

If one had to define what the purpose of modeling a dynamical system is, one could reasonably say it is describing how a set of variables of interest, call them $w$, evolve as a function of time. If we indicate by $\mathbb{T}$ the time axis of interest (typically $\mathbb{T}=\mathbb{R}$ for continuous time models and $\mathbb{T}=\mathbb{Z}$ for discrete time ones), and by $\mathbb{W}$ the space in which the variables of interest take on their values (e.g. $\mathbb{R}^{q}$ if there are $q$ real valued variables), then the $w$ 's are elements of $\mathbb{W}^{\mathbb{T}}$, with $\mathbb{W}^{\mathbb{T}}$ denoting the set of maps from $\mathbb{T}$ to $\mathbb{W}$. The model of the system tells us that only a subset of such trajectories can actually happen, namely the subset that complies with the laws of the system. We will indicate this set of admissible trajectories as $\mathfrak{B}$ and refer to it as the behavior of the dynamical system. 
Formalizing the above discussion, we define a dynamical system as a triple $\S=(\mathbb{W}, \mathbb{T}, \mathfrak{B})$, with $\mathbb{W}$ the signal space, $\mathbb{T}$ the time axis and $\mathfrak{B} \subseteq \mathbb{W}^{\mathbb{T}}$ the behavior of the system.

The above definition is the cornerstone of behavioral systems theory, and in essence it defines a model as an exclusion law, a rule that al ows us to pick a subset of feasible trajectories out of a set of possible ones. Given its crucial importance, we illustrate it with two examples.

1. Newton's second law imposes a restriction that relates the position $\vec{q}$ of a point mass to the force $\vec{F}$ acting on it. This relation is $\vec{F}=m \frac{d^{2}}{d t^{2}} \vec{q}$, with $m$ the mass. This is a dynamical system with $\mathbb{T}=\mathbb{R}, \mathbb{W}=\mathbb{R}^{3} \times \mathbb{R}^{3}$, and behavior $\mathfrak{B}$ consisting of all maps $t \in \mathbb{R} \mapsto(\vec{q}, \vec{F})(t) \in \mathbb{R}^{3} \times \mathbb{R}^{3}$ that satisfy $\vec{F}=m \frac{d^{2}}{d t^{2}} \vec{q}$.

2. Kepler's laws describe the possible motions of the planets in the solar system. They define a dynamical system with $\mathbb{T}=\mathbb{R}, \mathbb{V}=$ $\mathbb{R}^{3}$, and $\mathfrak{B}$ the set of maps $w: \mathbb{R} \rightarrow \mathbb{R}^{3}$ that satisfy the following laws. The paths $w$ must be ellipses in $\mathbb{R}^{3}$ with the sun (assumed in fixed position) at one of the foci, the radius vector from the sun to the planet must sweep out equal areas in equal time, and the ratio of the period of revolution around the ellipse to the major axis must be the same for all $w$ 's in $\mathfrak{B}$.

Classical notions such as linearity and time-invariance are also ir.troduced very naturally, starting from the above formal definition of a system. In particular, we talk about a linear system if $\mathbb{W}$ is a vector space and $\mathfrak{B}$ a linear subspace of $\mathbb{W}^{\mathbb{T}}$, and about a time-invariant one (assuming $\mathbb{T}=\mathbb{R}$ or $\mathbb{Z}$ ) if $\sigma^{t} \mathfrak{B}=\mathfrak{B}$ for all $t \in \mathbb{T}$, where $\sigma^{t}$ denotes the $t$-shift defined by $\left(\sigma^{t} f\right)\left(t^{\prime}\right):=f\left(t^{\prime}+t\right), t^{\prime} \in \mathbb{R}$.

\section{DIFFERENTIAL SYSTEMS}

As discussed in the above section, when it comes to modeling a dynamical system, what we are really after is the behavior $\mathfrak{B}$, the set of admissible trajectories. Of course such a set can be described in many possible ways, for example through differential equations as in Newton's second law, or through formal descriptions, such as in Kepler's laws. It is therefore conceptually misleading to identify the idea of a system with that of a set of differential equations, because, as we have pointed out, equations are just one of many possible instruments that can be used to specify behaviors. As further examples, think of finite state automata whose behavior is typically described graphically, or non-linear electronic components that are often described by graphs in the I-V plane. 
Although identifying systems with equations is not appropriate, the class of systems whose behavior is specified by differential equations deserves special attention, because it plays such a prominent role in physical and engineering applications. We define a differential system as a system with $\mathbb{T}=\mathbb{R}$, whose behavior $\mathfrak{B}$ consists of all solutions of a set of differential equations of the form

$$
f\left(t, w, \frac{d}{d t} w, \ldots, \frac{d^{L}}{d t^{L}} w\right)=0 .
$$

Of even greater interest to us is the subclass of differential systems for which $\mathbb{W}$ is a finite dimensional vector space, and the defining equations are not only linear but also the variable $t$ does not appear explicitly in the above equation. In this case we talk about a linear time-invariant differential system. If $\mathbb{W}$ is $q$-dimensional, say, the behavior is then specified as all solutions to

$$
R_{0} w+R_{1} \frac{d}{d t} w+\cdots+R_{L} \frac{d^{L}}{d t^{L}} w=0,
$$

with $R_{i} \in \mathbb{R}^{p \times q}, i=0,1, \ldots, L$, where $p$ denotes the number of rows in the above system. Notice how algebraic constraints (i.e. differential equations of order 0 ) are automatically included in this class. To avoid technical issues, in the following we regard $\mathfrak{B}$ as the set of $\mathfrak{C}^{\infty}$ solutions of the above set of equations, in other words we take $\mathfrak{B} \subseteq \mathfrak{C}^{\infty}\left(\mathbb{R}, \mathbb{R}^{q}\right)$.

To the above system of differential equations, we can associate in a natural way the polynomial matrix $R(\xi)=R_{0}+R_{1} \xi+\cdots+R_{L} \xi^{L} \in$ $\mathbb{R}[\xi]^{p \times q}$, the elements of the matrix being polynomials in $\xi \in \mathbb{R}$. Given this association, we often write the set of equations as

$$
R\left(\frac{d}{d t}\right) w=0 .
$$

For obvious reasons, we may refer to the above as a kernel representation of the behavior of our linear time-invariant differential system, and write $\mathfrak{B}=\operatorname{ker}\left(R\left(\frac{d}{d t}\right)\right)$.

If we are given a linear subspace $V \subseteq \mathbb{R}^{q}$, we know from basic linear algebra that we can always find a matrix $R$ such that $V=\operatorname{ker}(R)$; we also know that such a matrix is not uniquely defined. Something very similar happens when looking at kernel representations of linear differential behaviors, which are subspaces of $\mathfrak{C}^{\infty}\left(\mathbb{R}, \mathbb{R}^{q}\right)$; in order to investigate this aspect we first recall the definition of a module over the polynomial ring $\mathbb{R}[\xi]$.

The module spanned by a set $v_{1}, \ldots, v_{p} \in \mathbb{R}^{n}[\xi]$ of polynomial vectors, denoted by $\left\langle v_{1}, \ldots, v_{p}\right\rangle$, is defined as the set of all linear combinations 
with polynomial coefficients of the given vectors. Thus we have

$$
\left\langle v_{1}, \ldots, v_{p}\right\rangle=\left\{\sum_{i=1}^{p} h_{i} v_{i}: h_{i} \in \mathbb{R}[\xi]\right\} \subseteq \mathbb{R}^{n}[\xi]
$$

Further, $\mathbb{R}^{n}[\xi]$ itself is a module, trivially obtained as $\left\langle e_{1}, \ldots, e_{n}\right\rangle$ with $e_{i}$ the $i$-th unit vector. Modules of the form $\left\langle v_{1}, \ldots, v_{p}\right\rangle \subseteq \mathbb{R}^{n}[\xi]$ are submodules of $\mathbb{R}^{n}[\xi]$.

The set of generators of such a submodule is not unique. In other words there can exist elements $u_{1}, \ldots, u_{r} \in \mathbb{R}^{n}[\xi]$ such that $\left\langle v_{1}, \ldots, v_{p}\right\rangle$ $=\left\langle u_{1}, \ldots, u_{r}\right\rangle$. Because in general $p \neq r$, the cardinality of the generating set of a given module is also not uniquely determined. The minimal cardinality is unique, however; in other words, for any submodule $\mathfrak{M} \subseteq \mathbb{R}^{n}[\xi]$, there is a greatest integer $c$ such that any generating set of the given submodule must contain at least $c$ elements. Generating sets with exactly $c$ elements are called minimal generating sets for $\mathfrak{M}$.

Given a polynomial matrix $R \in \mathbb{R}^{p \times q}[\xi]$, we will now indicate by $\langle R\rangle$ the submodule spanned by its rows. It turns out that, if $R^{\prime}$ is also a polynomial matrix with $q$ columns, then

$$
\mathfrak{B}=\operatorname{ker}\left(R\left(\frac{d}{d t}\right)\right)=\operatorname{ker}\left(R^{\prime}\left(\frac{d}{d t}\right)\right) \Leftrightarrow\langle R\rangle=\left\langle R^{\prime}\right\rangle
$$

In other words, any behavior will admit many different kernel representations, but is associated with one and only one submodule of $\mathbb{R}^{q}[\xi]$, namely the module generated by the rows of one, and therefore all, of its possible kernel representations.

This non-uniqueness in the representation of a behavior is a consequence of our definition of a system as a set of trajectories, rather than as a set of equations. It also has practical relevance, because it enables us to use in each situation the representation which we find most appropriate for the purpose at hand.

The non-uniqueness in the cardinality of generating sets for modules implies not only that several matrices $R$ satisfy $\mathfrak{B}=\operatorname{ker}\left(R\left(\frac{d}{d t}\right)\right)$, but also that the row dimension of $R$ is not uniquely defined (i.e. the number of equations we need to specify a behavior is not unique). The above discussion, however, provides a minimal number of rows, say $c$, that such a matrix must contain. Any $R \in \mathbb{R}^{c \times q}[\xi]$ such that $\mathfrak{B}=\operatorname{ker}\left(R\left(\frac{d}{d t}\right)\right)$ will be called a minimal representation of $\mathfrak{B}$. The minimal representations correspond to polynomial matrices $R$ which are of full row rank over the ring $\mathbb{R}[\xi]$ (that is, $R \in \mathbb{R}^{c \times q}[\xi]$ has a nonsingular $c \times c$ submatrix). 


\section{MODELING THE INFLUENCE OF THE ENVIRONMENT}

When modeling systems, we will usually be dealing with "open" systems. This means that the systems interact with the environment around them, so the given model includes some freedom in the $w$ 's for the influence of the environment. From the mathematical point of view, this will show up in the fact that, if $\mathfrak{B}=\operatorname{ker}\left(R\left(\frac{d}{d t}\right)\right)$, and if $R \in \mathbb{R}^{p \times q}[\xi]$ is a full row rank (equivalently, a minimal) representation of $\mathfrak{B}$, then $p<q$. In other words the system is underdetermined, having more variables than equations.

A special case of the situation described above is obtained by looking at systems in the traditional input-output form, which are described by the equations

$$
P\left(\frac{d}{d t}\right) y=Q\left(\frac{d}{d t}\right) u
$$

with $P$ square, $\operatorname{det}(P) \neq 0$, and $P^{-1} Q$ a matrix of proper rational functions. Such systems correspond in the notation (1) to $R=\left[\begin{array}{ll}P & -Q\end{array}\right]$ and $w=\left(\begin{array}{l}y \\ u\end{array}\right)$. It can be shown that the $u$ 's are free in (2), meaning that, for any given $u$, there exists a $y$ that satisfies the equations (2). In addition, the $y$ 's are bounded, meaning that they are uniquely specified by $u$ and by the initial values $y(0), \frac{d}{d t} y(0), \ldots$.

\section{SIMULATING TRAJECTORIES}

As discussed above we are dealing mainly with underdetermined systems of equations. Suppose now that we are interested in simulating a possible system trajectory, in other words in reproducing one solution of the system of equations (1). It is necessary to deal with the underdetermination of the original system. A very general way of doing so is by specifying an additional set of equations of the form

$$
U\left(\frac{d}{d t}\right) w=V\left(\frac{d}{d t}\right) f
$$

with $f \in \mathfrak{C}^{\infty}\left(\mathbb{R}, \mathbb{R}^{f}\right)$. Typically, the choice of $U, V$ and $f$ corresponds to fixing the external influences that act on the system. As a special case of additional equations (3), we may choose $U=\left[\begin{array}{ll}0 & I\end{array}\right]$ and $V=I$. It follows from $w=\left(\begin{array}{l}y \\ u\end{array}\right)$ that (3) takes up the freedom in $u$ by the classical assignment $u=f$.

Another possibility is that one might want or have to impose a set of conditions that the system variables and their derivatives should satisfy 
at a given instant in time, say $t=0$. The conditions may take the form

$$
S\left(\frac{d}{d t}\right) w(0)=a
$$

where $a$ is a given real vector of suitable dimension.

Thus the problem becomes one of first investigating existence and uniqueness of a solution $w$, which satisfies both the system equations (1) and the extra constraints (3) and (4), and then providing an algorithm to compute a (unique) solution. In the next pages we shall address the first issue in detail and overlook the second one.

Notice that, by setting $K=\left(\begin{array}{c}R \\ U\end{array}\right)$ and $J=\left(\begin{array}{c}0 \\ V\end{array}\right)$, equations (1) and (3) can be written together as $K\left(\frac{d}{d t}\right) w=J\left(\frac{d}{d t}\right) f$. Slightly generalizing the situation described above, we will therefore look at the following problem. We are given polynomial matrices $K \in \mathbb{R}[\xi]^{p \times q}, S \in$ $\mathbb{R}[\xi]^{s \times q}, J \in \mathbb{R}[\xi]^{p \times m}$, a real vector $a \in \mathbb{R}^{s \times 1}$ and a function vector $f \in$ $\mathfrak{C}^{\infty}\left(\mathbb{R}, \mathbb{R}^{m}\right)$. These matrices and vectors provide the following system of differential equations with initial conditions:

$$
\left.\begin{array}{l}
K\left(\frac{d}{d t}\right) w=J\left(\frac{d}{d t}\right) f \\
S\left(\frac{d}{d t}\right) w(0)=a
\end{array}\right\} .
$$

The question is to determine conditions under which there exists a solution $w \in \mathfrak{C}^{\infty}\left(\mathbb{R}, \mathbb{R}^{q}\right)$ of these equations. The question is answered by the following theorem, already presented in [2].

Theorem $1:$ Let $K \in \mathbb{R}[\xi]^{p \times q}, S \in \mathbb{R}[\xi]^{s \times q}, J \in \mathbb{R}[\xi]^{p \times m}, a \in \mathbb{R}^{s \times 1}$ and $f \in \mathfrak{C}^{\infty}\left(\mathbb{R}, \mathbb{R}^{m}\right)$ be given. The system of differential equations with initial conditions (5) has a solution $w \in \mathfrak{C}^{\infty}\left(\mathbb{R}, \mathbb{R}^{q}\right)$ if and only if the data have the two properties

$$
\begin{aligned}
& \text { 1: } n \in \mathbb{R}^{1 \times p}[\xi] \text { and } n K=0 \Rightarrow n\left(\frac{d}{d t}\right) J\left(\frac{d}{d t}\right) f=0, \\
& \text { 2: } \quad \ell \in \mathbb{R}^{1 \times s}, \quad b \in \mathbb{R}^{1 \times p}[\xi] \text { and } \ell S=b K
\end{aligned}
$$

$$
\Rightarrow \ell a=\left(b\left(\frac{d}{d t}\right) J\left(\frac{d}{d t}\right) f\right)(0) .
$$

The first of these conditions states that, in order to have a solution $w$ to $K\left(\frac{d}{d t}\right) w=J\left(\frac{d}{d t}\right) f$ for a given $f$, any differential relationships which hold for the rows of $K$ must also hold for the corresponding components 
of the vector $J\left(\frac{d}{d t}\right) f$. Moreover, the other condition states that, if a solution satisfies $S\left(\frac{d}{d t}\right) w(0)=a$, then, whenever a linear combination of the left hand side of the initial conditions can be written as a consequence of the left hand side of the equations, then the right hand side of the initial conditions must be in the same way a consequence of the right hand side of the equations. The first condition, therefore, expresses consistency of the set of equations $K\left(\frac{d}{d t}\right) w=J\left(\frac{d}{d t}\right) f$, while the second one expresses consistency of the initial conditions with respect to the given equations. Both conditions can be seen as generalizing the well known rank condition for solvability of systems of algebraic equations $A x=y$, say.

A MATLAB pseudocode will be given at the end of this section that sketches an algorithm that checks these conditions. It requires the following useful concepts.

- For any $K \in \mathbb{R}[\xi]^{p \times q}$, the annihilators for the rows of $K$ are defined to be the elements of the set

$$
\mathfrak{H}_{K}=\left\{n \in \mathbb{R}^{1 \times p}[\xi]: n K=0\right\} .
$$

Such a set is a submodule of $\mathbb{R}^{p}[\xi]$; in algebraic literature it is called the syzygy module of the rows of $K$ (see $[4,1]$ ). One can always find a polynomial matrix $N$ such that its rows generate $\mathfrak{H}_{K}$, in other words such that $\mathfrak{H}_{K}=\langle N\rangle$. In [1] an algorithm is presented which constructs a suitable $N$ from $K$; such an algorithm is part of most computer algebra packages; in our pseudocode we assume that a procedure SYZYGY is available which performs this computation. Condition 1 of the above theorem can then be checked by verifying $N\left(\frac{d}{d t}\right) J\left(\frac{d}{d t}\right) f=0$.

- Given $K \in \mathbb{R}[\xi]^{p \times q}$, we define its highest row coefficient matrix $K_{h c}$ to be the real matrix whose $i$-th row contains the coefficients of the highest power of $\xi$ that occurs in the $i$-th row of $K$. For example,

$$
K=\left[\begin{array}{cc}
3 \xi^{2}+\xi+1 & 2 \\
2 \xi & \xi+1
\end{array}\right] \Rightarrow K_{h c}=\left[\begin{array}{ll}
3 & 0 \\
2 & 1
\end{array}\right]
$$

The matrix $K$ is defined to be row proper if the rows of $K_{h c}$ are linearly independent. If $K$ is not row proper, its row proper form is defined to be any matrix $K^{\prime}$ such that the modules $\langle K\rangle$ and $\left\langle K^{\prime}\right\rangle$ are the same, and such that $K^{\prime}$ is row proper. Of course $K^{\prime}$ is not uniquely defined, but any row proper form can be obtained from another by taking linear combinations of the rows. For example, 


$$
\text { if } \begin{aligned}
& K= {\left[\begin{array}{cc}
\xi^{2}+\xi+1 & \xi^{2} \\
\xi & \xi+1
\end{array}\right] \text {, then } } \\
& K_{1}^{\prime}=\left[\begin{array}{cc}
\xi+1 & -\xi \\
\xi & \xi+1
\end{array}\right] \text { and } K_{2}^{\prime}=\left[\begin{array}{cc}
2 \xi+1 & 1 \\
\xi & \xi+1
\end{array}\right]
\end{aligned}
$$

are both row proper forms of $K$. Because $\langle K\rangle=\left\langle K^{\prime}\right\rangle$, it follows that there exists a polynomial matrix $U$ such that $K^{\prime}=U K$. Our pseudocode uses a procedure $\left[K^{\prime}, U\right]=\operatorname{ROWPROP}(K)$ that returns a row proper form of $K$ and the transformation matrix $U$. Classical algorithms for doing so exist in the literature (e.g. in [5]).

- The leading monomial matrix $K_{l m}$ of $K \in \mathbb{R}[\xi]^{p \times q}$ is obtained by taking for each row the leftmost occurence of the highest power of $\xi$ appearing in the given row. For example,

$$
K=\left[\begin{array}{ccc}
\xi^{2}+\xi+1 & 2 & 3 \xi^{2} \\
4 & 2 \xi+1 & \xi
\end{array}\right] \Rightarrow K_{l m}=\left[\begin{array}{ccc}
\xi^{2} & 0 & 0 \\
0 & 2 \xi & 0
\end{array}\right]
$$

A matrix $P$ with $q$ columns is said to be reduced with respect to $K$ if there exists no polynomial matrix $V$ such that $P_{l m}=V K_{l m}$. For example, the vector $P=\left[\begin{array}{lll}\xi & 0 & 0\end{array}\right]$ is reduced with respect to the above $K$ while $P=\left[\begin{array}{lll}0 & \xi^{2} & 0\end{array}\right]$ is not.

Given any two polynomial matrices $K$ and $S$ with the same number of columns, a division algorithm can be designed that generates a quotient matrix $B$ and a remainder matrix $P$ such that $S=P+$ $B K$, where $P$ is reduced with respect to $K$. Our pseudocode uses a procedure $[B, P]=\operatorname{DIVIDE}(S, K)$, which returns the quotient and remainder matrix of the division of $S$ by $K$. An algorithm to perform this kind of division is described in [1], using generic orderings of vector polynomials that are more generic than our concept of leading monomials. Actually, all we are doing in this section could be recast in the more abstract language of canonical forms for polynomial matrices as in [2], but we prefer not to do so.

- Let $K^{\prime}$ be a row proper form of $K$ and let $S$ be as in the previous paragraph. Using the division algorithm we can find a quotient matrix $B^{\prime}$ and a remainder matrix $P$ such that $S=P+B^{\prime} K^{\prime}$. Because $K^{\prime}=U K$ for some matrix $U$, these remarks imply $S=$ $P+B K$ with $B=B^{\prime} U$. We note, however, that the $P$ and $B$ obtained in this way are not necessarily the same as those that would occur if we divided $S$ by $K$. This is a crucial and complex issue, very well addressed in $[4,1]$, and which reasons of space force us to overlook. 
- As a consequence of having divided by a row proper form of $K$, it can now be shown that the remainder matrix $P$ has the property that, given a real vector $\ell \in \mathbb{R}^{1 \times s}$, there exists a polynomial vector $b \in \mathbb{R}^{1 \times p}[\xi]$ such that $\ell S=b K$ if and only if $\ell P=0$; in this case we have $b=\ell B$, where $B$ is defined above. The set $C H_{P}$ of constant annihilators of the rows of $P$ is defined as:

$$
C H_{P}=\left\{\ell \in \mathbb{R}^{1 \times s}: \ell P=0\right\},
$$

which is a linear subspace of $\mathbb{R}^{s}$. Therefore standard linear algebra techniques allow us to build a matrix $L$ whose rows span $C H_{P}$. In our pseudocode we assume the availability of a procedure ANNIHIL that constructs such a matrix. Thus, remembering $b=\ell B$, the checking of condition 2 in the above theorem is reduced to a linear algebra problem, namely the computation of $L$ and the verification of $L a=\left(L B\left(\frac{d}{d t}\right) J\left(\frac{d}{d t}\right) f\right)(0)$.

With these remarks at hand, we can now sketch the desired algorithm:

Solv=SOLVABILITY $(K, J, S, f, a)$;

Solv=0;

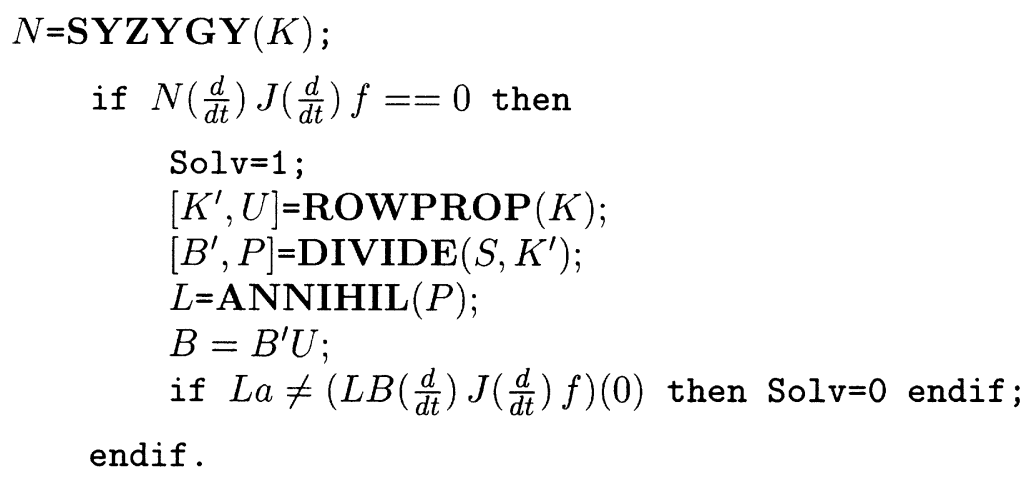

The procedure returns Solv $=0$ if the problem has no solution, Solv=1 otherwise.

We close this section by showing how the classical Cauchy problem fits into our framework.

Example 2 : Consider the first order system:

$$
\left.\begin{array}{l}
\dot{x}=A x+B f \\
x(0)=a
\end{array}\right\}
$$

which corresponds, in our notation, to $K=\xi I-A, S=I$ and $J=B$. Because $K$ is square and non-singular, the set $\mathfrak{H}_{K}$ of annihilators of the 
rows of $K$ contains only the zero vector, so $N=0$. We see that $K$ is row proper and that the division of $S$ by $K$ gives $P=S=I$ and $B=0$, so $C H_{P}$ also contains only the zero vector, i.e. $L=0$. Therefore, by applying Theorem 1 , we conclude the existence of a solution $x$ for arbitrary $f$ and $a$.

\section{OBSERVABILITY}

As announced in the introduction, the purpose of the final two sections of this paper is recasting the classical concepts of observability and controllability in the language we have used to describe dynamical systems; for the case of linear differential systems, we shall present algorithms which allow us to verify their properties in terms of the coefficients of the defining equations.

Traditionally, in the context of input/state/output systems of the form $\frac{d}{d t} x=f(x, u), y=h(x, u)$, observability is defined as the possibility of deducing, knowing the laws of the system, the state trajectory $x(\cdot)$ from observation of the input and output trajectories $u(\cdot)$ and $y(\cdot)$. In our context, however, no special variables such as the state show up in the definition of observability, which is now seen as the possibility of deducing the trajectories of a subset of the system variables $w$, given the laws of the system and observations of the remaining variables, which form a second subset. The first subset is often referred to as to-beobserved variables and denoted by $w_{2}$, while the latter is often referred to as observed variables and denoted by $w_{1}$.

Formally, let $\left(\mathbb{T}, \mathbb{W}_{1} \times \mathbb{W}_{2}, \mathfrak{B}\right)$ be a dynamical system for which the splitting of the signal space $\mathbb{W}$ into $\mathbb{W}_{1} \times \mathbb{W}_{2}$ corresponds to the separation of variables into observed and to-be-observed variables. We denote a typical element of the behavior by $w=\left(w_{1}, w_{2}\right) \in \mathfrak{B}$, and we define $w_{2}$ to be observable from $w_{1}$ if

$$
\left(w_{1}, w_{2}\right) \in \mathfrak{B} \quad \text { and } \quad\left(w_{1}, w_{2}^{\prime}\right) \in \mathfrak{B} \quad \Rightarrow \quad w_{2}=w_{2}^{\prime} .
$$

In the case when $\mathfrak{B}$ is a linear behavior, this is equivalent to the condition

$$
\left(0, w_{2}\right) \in \mathfrak{B} \quad \Rightarrow \quad w_{2}=0 .
$$

When discussing observability for $\mathfrak{B}=\operatorname{ker}\left(R\left(\frac{d}{d t}\right)\right)$, it is convenient to partition $R$ into two parts that correspond to its action on the $w_{1}$ and $w_{2}$ subsets. Therefore we rewrite the behavioral equations $R\left(\frac{d}{d t}\right) w=0$ as

$$
N\left(\frac{d}{d t}\right) w_{1}=M\left(\frac{d}{d t}\right) w_{2}
$$


Before giving conditions for observability we recall two definitions. A matrix $G \in \mathbb{R}^{p \times p}[\xi]$ is said to be unimodular if it admits a polynomial inverse, in other words if there exists a matrix $F$ such that $F G=G F=$ $I$; this is equivalent to $\operatorname{det}(G)=g \in \mathbb{R} \backslash\{0\}$. A polynomial matrix $R \in \mathbb{R}^{p \times q}[\xi]$ is said to be left prime if $R=G R^{\prime}$, with $G \in \mathbb{R}^{p \times p}[\xi]$ and $R^{\prime} \in \mathbb{R}^{p \times q}[\xi]$, implies that $G$ is unimodular; the definition of right primeness is analogous.

We can now state a theorem (see [3]) that presents equivalent conditions for observability.

Theorem 3 : Let $\mathfrak{B}$ be the set

$$
\mathfrak{B}=\left\{w=\left(w_{1}, w_{2}\right) \in \mathfrak{C}^{\infty}\left(\mathbb{R}, \mathbb{R}^{q}\right): N\left(\frac{d}{d t}\right) w_{1}=M\left(\frac{d}{d t}\right) w_{2}\right\},
$$

and assume $M \in \mathbb{R}^{p \times \ell}[\xi]$. Then the following properties are equivalent:

$1 w_{2}$ is observable from $w_{1}$,

2 There exist unimodular matrices $U$ and $V$ such that

$$
U M V=\left[\begin{array}{l}
I \\
0
\end{array}\right]
$$

$3 \operatorname{rank}(M(\lambda))=\ell$ for any $\lambda \in \mathbb{C}$,

$4 M$ is right prime,

5 The rows of $M$ span the full module $\mathbb{R}^{\ell}[\xi]$.

Condition 5 of Theorem 3 can be restated as follows: consider the set of row vectors of degree 0 contained in the module spanned by the rows of $M$; such a set is obviously an $\mathbb{R}$-vector space contained in $\mathbb{R}^{\ell}$, and, in order for $M$ to be right prime, it must be $\mathbb{R}^{\ell}$ itself. We now sketch an algorithm for investigating such a condition. It recursively builds a generating set for the above vector space, and checks whether the dimension of the space is $\ell$. We employ the following notation.

- Standard MATLAB notation will be used to indicate the rows and columns of a matrix, e.g. $M(i,:)$ is the $i$-th row of $M$, and $M(i: j,:)$ are rows $i$ to $j$ of $M$.

- $M^{0}$ denotes the set of rows of the matrix $M$ that have degree 0 . For example

$$
M=\left[\begin{array}{cc}
\xi^{2}+\xi+1 & 2 \\
1 & 1
\end{array}\right] \quad \Rightarrow \quad M^{0}=\left[\begin{array}{ll}
1 & 1
\end{array}\right]
$$


- $d_{i}$ is the degree of the $i$-th row of $M$, so in the above example we have $d_{1}=2$ and $d_{2}=0$.

- $M_{h c} \in \mathbb{R}^{p \times \ell}$ is the highest row coefficient matrix of $M$ as defined in the preceding section. Further, $M_{h p} \in \mathbb{R}^{p \times \ell}[\xi]$ is the highest row power matrix of $M$, meaning that, for $i=1,2, \ldots, p$, the row $M_{h p}(i,:)$ contains just the highest power of $\xi$ in $M(i,:)$ with its coefficients. Therefore $M_{h p}(i,:)$ is the product $\xi^{d_{i}} M_{h c}(i,:)$, and in the above example we have

$$
M_{h c}=\left[\begin{array}{ll}
1 & 0 \\
1 & 1
\end{array}\right] \text { and } M_{h p}=\left[\begin{array}{cc}
\xi^{2} & 0 \\
1 & 1
\end{array}\right] .
$$

- $M=\operatorname{Order}(M)$ is a procedure that permutes the rows of $M$ into decreasing row degree order. For example

$$
M=\left[\begin{array}{cc}
\xi & 1 \\
\xi^{2}+\xi+1 & 2 \\
1 & 1
\end{array}\right] \Rightarrow \operatorname{Order}(M)=\left[\begin{array}{cc}
\xi^{2}+\xi+1 & 2 \\
\xi & 1 \\
1 & 1
\end{array}\right]
$$

- Let the $p \times \ell$ matrix $M$ be ordered by $\operatorname{Order}(M)$, and then let $M_{h c}$ be formed. For any integer $i$ between 1 and $p-1$, standard linear algebra allows us to find, if it exists, a non-zero real vector $n$ such that $M_{h c}(i,:)=n M_{h c}(i+1: p,:)$. For example,

$$
M=\left[\begin{array}{cc}
\xi^{2}+\xi+1 & \xi^{2}+2 \\
\xi & 1 \\
0 & 1
\end{array}\right] \Rightarrow M_{h c}(1,:)=n M_{h c}(2: 3,:)
$$

for $n=\left[\begin{array}{ll}1 & 1\end{array}\right]$. Given such an $n$, the function $h=\operatorname{polann}(n, M)$ returns a polynomial vector $h$ such that $M_{h p}(i,:)=h M_{h p}(i+$ $1: p,:)$. In the above case, for example, $\operatorname{polann}(n, M)$ returns $h=\left[\begin{array}{ll}\xi & \xi^{2}\end{array}\right]$. To build $h$ from $n$ is straightforward, because, for $k=i+1, \ldots, p$, the element of $h$ that multiplies the $k$-th row of $M_{h p}$ is the corresponding element of $n$ times $\xi^{d_{i}-d_{k}}$.

- $M=\operatorname{Eliminate}(M, i)$ is a procedure that removes the $i$-th row of a matrix $M$. For example, if

$$
M=\left[\begin{array}{cc}
\xi^{2}+\xi+1 & \xi^{2}+2 \\
\xi & 1 \\
0 & 1
\end{array}\right]
$$

then Eliminate $(M, 2)$ returns

$$
M=\left[\begin{array}{cc}
\xi^{2}+\xi+1 & \xi^{2}+2 \\
0 & 1
\end{array}\right]
$$


- Let $m$ be a polynomial row vector of degree $d_{m}$ and length $p$, and let $M \in \mathbb{R}^{p \times l}[\xi]$ be a matrix ordered as by Order; moreover, let $j$ be such that the degree of $M(i,:)$ is less than or equal to $d_{m}$ for $i \geq j$ and greater than $d_{m}$ for $i \leq j-1$. Then $[M, j]=\operatorname{Insert}(M, m)$ is a procedure that replaces the matrix $M$ by the matrix with the $p+1$ rows $(M(1: j-1,:), m, M(j: p,:))$, where, as usual, $p$ is the number of rows of the original $M$. The procedure also returns $j$, which is the row index of $m$ in the new matrix. For example, if

$$
M=\left[\begin{array}{cc}
\xi^{2}+\xi+1 & \xi^{2}+2 \\
1 & 1
\end{array}\right] \text { and } m=\left[\begin{array}{ll}
\xi+1 & \xi
\end{array}\right]
$$

then $\operatorname{Insert}(M, m)$ returns

$$
M=\left[\begin{array}{cc}
\xi^{2}+\xi+1 & \xi^{2}+2 \\
\xi+1 & \xi \\
1 & 1
\end{array}\right] \quad \text { and } \quad j=2
$$

We can now sketch (in MATLAB pseudo-code) our procedure for checking right primeness (RPR) of a matrix $M$, and hence observability of a differential system.

$[M, \mathrm{obs}]=\mathbf{R P R}(M)$;

$$
\begin{aligned}
& M=\operatorname{Order}(M) \text {; } \\
& \text { obs }=\left(\operatorname{rank}\left(M^{0}\right)==\ell\right) \text {; } \\
& p=\operatorname{rowdim}(M) \text {; } \\
& i=p \text {-rowdim }\left(M^{0}\right) \text {; } \\
& \text { while ( (not obs) and }(i \geq 1)) \text { do } \\
& \text { if ( } \exists \text { real } n \neq 0 \text { such that } \\
& \left.M_{h c}(i,:)=n M_{h c}(i+1: p,:)\right) \text { then } \\
& h=\operatorname{polann}(n, M) \text {; } \\
& m=M(i,:)-h M(i+1: p,:) \text {; } \\
& M=\operatorname{Eliminate}(M, i) \text {; } \\
& \text { if }(m \neq 0) \text { then } \\
& {[M, j]=\operatorname{Insert}(M, m) \text {; }} \\
& \text { if (degree }(m)==0 \text { ) then } \\
& \text { obs }=\left(\operatorname{rank}\left(M^{0}\right)==\ell\right) \text {; } \\
& i=j-1 \text {; }
\end{aligned}
$$




$$
\begin{aligned}
& \qquad \text { else } i=j \text { endif; } \\
& \text { else } p=p-1 \quad i=i-1 \text { endif; } \\
& \text { else } i=i-1 \text {; } \\
& \text { endif; }
\end{aligned}
$$

endwhile.

In the above algorithm obs is a boolean variable that tells us whether the matrix we are considering is right prime or not; we call this variable obs because of the relation between right primeness and observability explained in Theorem 3. As already discussed, right primeness is checked by verifying if a generating set for the vectors of degree 0 contained in the module spanned by the rows of $M$ has rank equal to $\ell$. After reordering $M$, we immediately perform a check to see whether the vectors of degree 0 in the original matrix are already sufficient to meet the requirement. If this is not the case, after setting $i=p-\operatorname{row} \operatorname{dim}\left(M^{0}\right)$, which gives the index of the first row from the bottom with degree higher than 0 , we enter the main while loop in which we try to generate additional constant vectors by taking combinations of rows of $M$. This is done by replacing a row $M(i,:)$ of degree higher than 0 by a polynomial combination of $M(i,:)$ and later rows of $M$, provided that the combination is of lower degree than $M(i,:)$ itself. Such a degree lowering is possible only if the highest coefficient vector of $M(i,:)$ is linearly dependent on the highest coefficient vectors of the rows of equal or lower degree. This condition is tested in the "if" statement by looking for a real vector $n \neq 0$ of suitable dimension such that $M_{h c}(i,:)=n M_{h c}(i+1: p,:)$. If such a dependence is found, then starting from $n$ we build the polynomial vector $h$ such that $M_{h p}(i,:)=h M_{h p}(i+1: p,:)$. Thus the polynomial vector $m=M(i,:)-h M(i+1: p,:)$ has degree lower than $M(i,:)$, as desired.

If such a degree lowering is found to be possible, we then eliminate $M(i,:)$. Further, when the new row vector $m$ is not zero, it is included in the matrix $M$ in the earliest position that maintains the degree ordering of Order. If the new vector has degree 0 , we check again if the right primeness condition is fulfilled; alternatively, the next iteration of the while loop will check whether the degree of this newly generated vector can itself be lowered (this is the reason for having $i=j$ ).

The algorithm ends if the condition for right primeness is verified (obs is true) or if no more lowering of degree is possible. When the condition $i<1$ occurs in the while statement, this means that we have considered all the rows in $M$, so there is no possibility of further lowering. Because 
at each step we are replacing a vector by one of lower degree, or are reducing the number of rows of $M$, the $i<1$ condition will always hold eventually. Therefore the stopping rule for the algorithm is well defined.

We see that the final matrix $M$ is an output argument of our procedure. This is not needed when the only output of interest is obs; it will, however, be handy in the next section, where $\mathbf{R P R}$ is a subprocedure of a procedure that checks controllability.

As an example of the above algorithm, we wish to show its application to the observability matrix for state space systems.

Example 4 : We consider the classical problem of deducing the state trajectory $x(\cdot)$, starting from observations of the input and output trajectories $u(\cdot)$ and $y(\cdot)$ in the state space system

$$
\left.\begin{array}{l}
\dot{x}=A x+B u \\
y=C x+D u
\end{array}\right\}
$$

Such a problem corresponds, in our formalism, to

$$
w_{1}=\left(\begin{array}{l}
u \\
y
\end{array}\right), \quad w_{2}=x \text { and } M=\left[\begin{array}{c}
-\xi I+A \\
C
\end{array}\right]
$$

where $M \in \mathbb{R}^{p \times \ell}[\xi]$ and $I$ is the $\ell \times \ell$ identity matrix.

The algorithm provides an efficient way of checking the classical rank conditions on the observability matrix $\left(\begin{array}{c}C \\ C A \\ \vdots \\ C A^{\ell-1}\end{array}\right)$. It can be shown that the rows of this matrix are vectors of degree 0 contained in the module spanned by the rows of $M$. Specifically, the rows of $C A$ can be expressed as $(\xi I) C+C(-\xi I+A)$, and are therefore polynomial linear combinations of degree 0 of the rows of $M$. By induction, one finds that the rows of $C A^{k}=(\xi I) C A^{k-1}+C A^{k-1}(-\xi I+A)$ are also 0 degree vectors in the module spanned by the rows of $M$. Further, the rows of the observability matrix are actually a generating set for the space of all 0 degree vectors spanned by the rows of $M$; this remark follows easily from the fact that $A^{\ell}$ is linearly dependent on $I, A, \ldots A^{\ell-1}$, so no independent row vectors would be added by considering $C A^{k}$ for $k \geq \ell$.

Checking that the observability matrix has rank $\ell$ is therefore equivalent to verifying that a generating set for the space of vectors of degree 0 contained in the module spanned by the rows of $M$ has rank $\ell$. As discussed above, this task is done by our algorithm in an efficient way. For example, consider the case when $C$ is a non-singular matrix. Then 
observability is found immediately without computing the rest of the observability matrix; in this case, in fact, our algorithm stops without even entering the main "while" loop.

\section{CONTROLLABILITY}

In the context of state space systems, the concept of controllability addresses the possibility of reaching any final state starting from any initial state in finite time. The state system $\frac{d}{d t} x=f(x, u)$ is said to be controllable if, for any initial state $x_{0}$ and any final state $x_{f}$, there exists an input function $u$ and a time $T$ such that the solution to $\frac{d}{d t} x=f(x, u)$ with initial condition $x(0)=x_{0}$ yields $x(T)=x_{f}$.

As in the case of observability, we now give a definition of controllability which relies only on properties of the system's trajectories, and not on specific properties of special variables chosen to represent it, namely state variables in the above example.

In particular, if $\mathfrak{B}$ is a continuous-time, time invariant behavior, we define it to be controllable if, for any two trajectories $w_{1}, w_{2} \in \mathfrak{B}$, there exists $t_{1} \geq 0$ and a third trajectory $w \in \mathfrak{B}$ such that

$$
w(t)= \begin{cases}w_{1}(t), & t \leq 0 \\ w_{2}\left(t-t_{1}\right), & t \geq t_{1}\end{cases}
$$

The intuition behind this definition is that, for a behavior to be controllable, one must be able to connect any admissible "undesired" past trajectory to any admissible "desired" future one, through suitable steering.

The concepts of controllability and observability are of central importance in systems theory, in particular in controller design and stabilization $[10,5]$, and in observer design and filtering [5], respectively. Very roughly speaking, controllability implies that a system can be stabilized by control. More precisely, the system, viewed as a plant, can be interconnected with a controller such that all solutions that satisfy the equations of both the plant and the controller go to zero (at an arbitrarily fast rate) as time goes to infinity. Observability in turn implies the existence of a signal processor that deduces, in a suitable way, the tobe-estimated variables (the $w_{2}$ 's in the definition of observability) from the observed variables (the $w_{1}$ 's in the definition of observability).

Again, as for observability, we now concentrate on the case of linear differential systems $\mathfrak{B}=\operatorname{ker}\left(R\left(\frac{d}{d t}\right)\right)$. We seek conditions that are equivalent to controllability in terms of the polynomial matrix $R$.

Unfortunately, we have to examine two situations separately: the case in which $R$ is of full row rank (equivalently, it provides a minimal kernel 
representation of $\mathfrak{B}$ ) and the case in which it is not. Notice that in the observability case any attempt to observe "too many" variables (i.e. $M$ does not have full column rank) is excluded by Theorem 3, whereas using "too many" equations to describe $\mathfrak{B}$ (i.e. $R$ does not have full row rank) may allow controllability of $\mathfrak{B}$, even though, as we shall see, the conditions on $R$ become less elegant.

The above remark may seem strange to anyone used to the old principle that controllability and observability are dual concepts; in the setting we are working in we abandon this adage. Indeed, our definitions show that, while controllability is essentially a property of the behavior, observability also depends on the choice of observed and to-be-observed variables. The fact that technical conditions for checking observability and controllability often turn out to be "dual" (in some sense) should not make us forget the fundamental difference just mentioned.

We now state two theorems (see [3]) which present conditions for controllability in terms of the polynomial matrix $R$. The first result applies to the general case.

Theorem 5 : The following are equivalent:

$1 \mathfrak{B}=\operatorname{ker}\left(R\left(\frac{d}{d t}\right)\right)$ is controllable,

2 There exist unimodular matrices $U$ and $V$ such that

$$
U R V=\left[\begin{array}{ll}
I & 0 \\
0 & 0
\end{array}\right]
$$

$3 \operatorname{rank}(R(\lambda))$ is independent of $\lambda$ for any $\lambda \in \mathbb{C}$,

4 If $N(\xi)$ is a minimal generating set for the module spanned by the columns of $R$, then $N(\xi)$ is a right prime matrix.

In the case when $R$ has full row rank, much more can be said, this time yielding a result which can be regarded as the "dual" of Theorem 3 .

Theorem 6 : Let $R(\xi) \in \mathbb{R}^{p \times q}[\xi]$ be a full row rank polynomial matrix. The following are equivalent:

$1 \mathfrak{B}=\operatorname{ker}\left(R\left(\frac{d}{d t}\right)\right)$ is controllable,

2 There exist unimodular matrices $U$ and $V$ such that $U R V=\left[\begin{array}{ll}I & 0\end{array}\right]$,

$3 \operatorname{rank}(R(\lambda))=p$ for any $\lambda \in \mathbb{C}$,

$4 R$ is left prime,

5 The columns of $R$ span the full module $\mathbb{R}^{p}[\xi]$. 
We now sketch an algorithm that assesses controllability for a given $\mathfrak{B}=\operatorname{ker}\left(R\left(\frac{d}{d t}\right)\right)$.

To begin with notice that a matrix is left prime if and only if its transpose is right prime. Therefore, given the algorithm of the preceding section, it is easy to build a procedure LPR which checks whether a matrix $R$ is left prime (equivalently whether $\operatorname{ker}\left(R\left(\frac{d}{d t}\right)\right)$ is controllable under the assumptions of Theorem 6$)$. We can use

\section{$[R, \operatorname{ctr}]=\operatorname{LPR}(R)$;}

$$
\begin{aligned}
& M=R^{T} ; \\
& {[M, \operatorname{ctr}]=\operatorname{RPR}(M) ;} \\
& R=M^{T} .
\end{aligned}
$$

We know, however, that left primeness is equivalent to controllability only when $R$ is a full rank matrix; to check controllability in the general case we have to verify the conditions of Theorem 5 . In order to sketch an algorithm that does so, we make two remarks.

1 A column proper form of a matrix $R$ is obtained just by transposing the row proper form of $R^{T}$, with row proper defined in Section 5 . If $\mathbf{L P R}$ returns ctr=false, then the matrix $R$ which is returned is very close to a column proper form of the original $R$. Indeed, if left primeness is not verified, than the algorithm stops when the highest column coefficient vectors of all columns with degree higher than 0 are linearly independent of the highest column coefficient vectors of the subsequent columns. For vectors of degree 0 , however, we always check their rank but not their independence, so they need not be linearly independent.

The columns with degree higher than 0 , therefore, already satisfy the property that defines the column proper form of a matrix $R$, so to get a column proper form it is sufficient to replace the degree 0 columns of the returned $R$ by a basis of the $\mathbb{R}$-vector space they generate. The procedure $R=\operatorname{COLPRP}(R)$ brings the returned $R$ into column proper form in this way.

2 If $R$ is in column proper form, then the number of its columns is equal to the rank of the original matrix $R$. Therefore, if this number is equal to the row dimension of $R$, then $R$ is of full row rank, so, by Theorem 6, the test performed by the call of $\mathbf{L P R}$ is necessary and sufficient for controllability.

Alternatively, if the rank is smaller than the row dimension of $R$, we apply Condition 4 of Theorem 5 to check controllability. In this case the column proper form is a minimal generating set for the 
module spanned by the columns of $R$. Therefore we need to verify that the column proper form is right prime in order to conclude controllability.

The above remarks provide the following algorithm for checking controllability.

$[R, \operatorname{ctr}]=\operatorname{CTRB}(R)$;

$[R, \operatorname{ctr}]=\mathbf{L P R}(R)$;

if (not ctr) then

$R=\operatorname{COLPRP}(R)$;

if $(\operatorname{rowdim}(R)>\operatorname{coldim}(R))$ then

$[R, \operatorname{ctr}]=\mathbf{R P R}(R)$;

endif ;

endif .

As for observability, it can be shown that our algorithm corresponds to the usual test on the controllability matrix, in the case of checking controllability of a state space system, where $R$ has the form $R=$ $\left(\begin{array}{ll}\xi I-A-B \\ -\end{array}\right)$. We are going to show how it applies a known test for controllability of systems described by a single differential equation.

Example 7 : Assume $R=\left(r_{1} r_{2}\right)$, where $r_{1}$ and $r_{2}$ are polynomials and $d_{1}=$ degree $\left(r_{1}\right) \geq d_{2}=$ degree $\left(r_{2}\right)$. By applying a division algorithm for polynomials, we can write $r_{1}=q_{2} r_{2}+r_{3}$ with $d_{2}=\operatorname{degree}\left(r_{2}\right)>d_{3}=\operatorname{degree}\left(r_{3}\right)$. Going through our algorithm we see that, after at most $d_{1}-d_{2}+1$ steps, it will yield $R=\left(r_{2} r_{3}\right)$. Similarly, we write $r_{2}=q_{3} r_{3}+r_{4}$, and after at most $d_{2}-d_{3}+1$ more steps it will provide $R=\left(\begin{array}{ll}r_{3} & r_{4}\end{array}\right)$. Thus our algorithm applies the classical Euclidean algorithm for computing the greatest common divisor of two polynomials. Therefore at the end of the calculation we will have $R=r$ with $r=\operatorname{GCD}\left(r_{1}, r_{2}\right)$, so the condition for controllability is equivalent to asking if $r$ is a constant, which is equivalent to $r_{1}$ and $r_{2}$ being coprime polynomials.

\section{CONCLUSIONS}

In this paper we have discussed some basic concepts of the behavioral approach to dynamical modelling. We have treated in particular the simulation question, i.e. the problem of adding the specification of externally given signals and internally given initial data, so that the sys- 
tem has a (unique) solution. Further, we elaborated on the notions of controllability and observability in this setting.

Our presentation has concentrated on lumped linear differential systems. Present work aims at extending these ideas to distributed and nonlinear systems, and to extending the range of applications, particularly in the area of $\mathcal{H}_{\infty}$ control and filtering.

\section{References}

[1] W. Adams and P. Loustanau (1994), An Introduction to Gröbner Bases, AMS.

[2] T. Cotroneo and J.C. Willems (1999), The initial value problem for high order linear differential systems, Proceedings 1999 European Control Conference, Karlsruhe, Germany.

[3] T. Cotroneo and J.C. Willems (1999), Controllability and observability of linear differential behaviors, Proceedings 38th Conference on Decision Control, Phoenix, USA.

[4] D. Cox, J. Little, and D. O'Shea (1997), Ideals, Varieties and Algorithms:An Introduction to Computational Algebraic Geometry and Commutative Algebra, 2nd edition, Springer Verlag.

[5] T. Kailath (1980), Linear Systems, Prentice Hall.

[6] H.K. Pillai and S. Shankar (1999), A behavioral approach to control of distributed systems, SIAM Journal on Control and Optimization, 37, pp. 388-408.

[7] J.W. Polderman and J.C. Willems (1998), Introduction to Mathematical Systems Theory: A Behavioral Approach, Springer Verlag.

[8] P. Rocha and J.C. Willems (1991), Controllability of 2-D systems, IEEE Transactions on Automatic Control, 36, pp. 413-423.

[9] J.C. Willems (1991), Paradigms and puzzles in the theory of dynamical systems, IEEE Transactions on Automatic Control, 36, pp. 259-294.

[10] J.C. Willems (1997), On interconnections, control, and feedback, IEEE Transactions on Automatic Control, 42, pp. 326-339. 